\title{
Las zonas periurbanas de las grandes metrópolis, clave de las políticas de reinserción de naturaleza para mejorar el entorno de vida de los ciudadanos
}

\section{THE PERI-URBAN AREAS OF METROPOLISES, KEY TO GREENING POLICIES FOR IMPROVING THE LIVING ENVIRONMENT OF CITIZENS}

\author{
AS ZONAS PERIURBANAS DAS GRANDES METRÓPOLES, CHAVE \\ PARA AS POLÍTICAS DE REINSERÇÃO DE NATUREZA PARA \\ MELHORAR O AMBIENTE DE VIDA DOS CIDADÃOS
}

Laurène Wiesztort ${ }^{1}$

Para citar este artículo: Wiesztort, L. (2020). Las zonas periurbanas de las grandes metrópolis, clave de las políticas de reinserción de naturaleza para mejorar el entorno de vida de los ciudadanos. Perspectiva Geográfica, 25(2) 102-122. https://doi.org/10.19053/01233769.100917
Recepción:

28 de noviembre de 2019

Evaluación:

10 de junio de 2020

Aprobación:

2 de julio de 2020

\section{Resumen}

Las ciudades cada vez más densas sustituyen los espacios naturales y agrícolas por la extensión urbana. Por eso, los habitantes expresan un malestar. A raíz de esta constatación, en 1950 aparecen las primeras preguntas sobre las ciudades, que analizan como temas fundamentales el desarrollo sostenible y el calentamiento global con el objetivo de generar investigaciones para mejorar el entorno urbano,

1 Doctora en Geografía, título otorgado por la Universidad De Artois, Arras, Francia. Además, forma parte del laboratorio Discontinuités, Universidad Lille Nord de France, Artois, EA 2468, France. Correo: wiesztort.l@hotmail.fr. 
haciéndolas más sostenibles y habitables. Las conclusiones son alarmantes y es por esa razón que están surgiendo nuevas políticas públicas de reinserción de naturaleza en las ciudades, pero ¿cómo lograrlo en ciudades densas? ¿Qué tipo de naturaleza? ¿Para quién? ¿En qué formas? Así, el objetivo de este artículo es inventariar, observar y criticar estos grandes proyectos para responder a esta problemática. La metodología se construyó con análisis de literatura y conceptos, y trabajos de campo para comprender la evolución de las políticas, los proyectos y sus desafíos desde los años 1950. Abordaremos los conceptos de cinturones verdes y luego de infraestructuras verdes, tramas verdes y azules, lineales originalmente diseñados para convertirlos en parques en el corazón de las metrópolis. Por último, nos hemos interesado en proyectos recientes, en particular dos, para generar una crítica.

Palabras clave: bienestar, ciudad habitable, espacio natural, medio ambiente, metrópolis, política pública.

\section{Abstract}

Increasingly dense cities have substituted natural and agricultural spaces for urban sprawl, and inhabitants have expressed their discomfort. As a result of this finding, the first questions about cities were brought up in 1950 to analyze sustainable development and global warming as fundamental issues for research in order to improve the urban environment and make cities more sustainable and livable. The conclusions are alarming and, therefore, new greening policies are emerging, but how to achieve greening in dense cities? What kind of nature? Where? For whom? This article intends to inventory, observe, and assess these large projects to tackle this problem. The method is based on literature and concept analyses and fieldwork to understand the evolution of policies, projects, and challenges since the 1950s. We address the concepts of green belts, green infrastructures, green and blue frames, and linear parks initially designed in the heart of metropolises. Finally, we review recent projects of our interest, mainly two.

Keywords: Well-being, livable city, natural space, environment, metropolis, public policy. 


\section{Resumo}

As cidades cada vez mais densas substituem os espaços naturais e agrícolas pela expansão urbana. Por isso, os moradores expressam um mal-estar. Como resultados dessa constatação, em 1950 surgiram às primeiras questões sobre as cidades, que analisam como questões fundamentais o desenvolvimento sustentável e o aquecimento global com o objetivo de gerar pesquisas para melhorar o ambiente urbano, tornando-as mais sustentáveis e habitáveis. As conclusões são alarmantes e é por isso que estão surgindo novas políticas públicas de reinserção da natureza nas cidades, mas como fazê-lo nas cidades densas? Que tipo de natureza? Para quem? De que maneiras? Assim, o objetivo deste artigo é inventariar, observar e criticar esses grandes projetos para responder a esta problemática. A metodologia foi construída com análise de literatura e conceitos, e trabalhos de campo para compreender a evolução das políticas, os projetos e seus desafios desde a década de 1950. Abordaremos os conceitos de cinturões verdes e posteriormente infraestruturas verdes, tramas verdes e azuis, lineais originalmente projetados para se tornarem parques no coração da metrópole. Finalmente, temos estado interessados em projetos recentes, em particular dois, para gerar uma crítica.

Palavras-chave: bem-estar, cidade habitável, espaço natural, meio ambiente, metrópole, políticas públicas.

\section{Introducción}

Con ciudades cada vez más densas en el mundo y los fenómenos de metropolización y extensión urbana (Gómez Mendoza, 2003), los espacios naturales en las ciudades son cada vez menos (Ruiz et al., 2015) o son difícilmente accesibles para muchos ciudadanos. En el corazón de estos territorios urbanos, la naturaleza está ausente o híbrida (fabricada, modificada por el ser humano) (Wiesztort, 2011).
Los habitantes de las ciudades expresan una falta de naturaleza. Desean estar en contacto con la naturaleza. Se habla de "malestar", en oposición a los numerosos estudios que demuestran la influencia positiva de la naturaleza en el bienestar y la salud de las personas (Ulrich, 1984; Björk et al., 2008; Park et al., 2009b; Grahn \& Stigsdotter, 2010; Raanaas et al., 2011; MacKerron \& Mourato, 2013; White et al., 2013; Bilal et al., 2016). Para atender esa ausencia, se han creado numerosas políticas públicas en las grandes metrópolis 
del mundo, pero la reinserción de naturaleza en la ciudad es difícil debido a la presión inmobiliaria. En ese contexto, las zonas periurbanas pueden desempeñar un papel importante. El espacio periurbano es el espacio intermedio entre las ciudades y el campo, compuesto de casas individuales rodeadas de jardines. En este espacio coexisten zonas edificadas y no edificadas donde se encuentran espacios naturales. El periurbano ha surgido, en sus diversas denominaciones, en los llamados países del norte de Europa para describir la renovación de las formas urbanas y de los modos de habitar en la periferia de los grandes centros urbanos (Aragau, 2018).

A continuación se analizarán algunos de esos proyectos. Así pues, se abordará la realización de dos parques lineales y sus evoluciones a lo largo del tiempo en las zonas periurbanas de dos grandes metrópolis: Lille (Francia) y Río de Janeiro (Brasil) para responder — desde un enfoque críticoa las siguientes cuestiones: ¿qué tipo de naturaleza y espacio natural quieren crear? ¿Para quién? ¿En qué formas?

La metodología de este trabajo de investigación se construye con análisis de literatura y conceptos, y trabajos de campo: uno que realizó la geógrafa Laurène Wiesztort en el territorio regional de Nord-Pas-de-Calais en 2011 sobre las expectativas específicas en términos de espacios naturales de los habitantes de la metrópolis Lille, y otro realizado en Río de Janeiro por la geógrafa doctorante N. Amorim, de la Universidad Federal de Bahía (Amorim \& Wiesztort, 2018). También se usarán referencias a otras investigaciones y publicaciones internacionales.

\section{La falta de naturaleza en las}

\section{grandes metrópolis mundiales}

\subsection{Constatación alarmante}

La ciudad es un espacio en el cual viven los ser humanos y juega un papel muy importante en la creación de una sociedad particular. Como subraya Cluzet en su obra $A u$ bonheur des villes (2002): se han convertido en el principal biotopo de los hombres. Así, deben ofrecer condiciones de vivienda adaptadas a las necesidades actuales y a la vez condiciones de bienestar. Por lo tanto, realizar un buen diseño de las ciudades y una buena organización del territorio urbano son asuntos muy importantes.

Hoy la urbanización no planificada genera problemas medioambientales que provocan un aumento de la contaminación urbana, temperaturas superiores en el centro de la ciudad, una mineralidad creciente (revestimientos de hormigón, piedra y ausencia de vegetación) y la desaparición de zonas verdes (Ruiz et al., 2015; Melé, 2004; Gómez Mendoza, 2003). Además, en otros contextos existen otras causas. Por ejemplo, en Colombia el desplazamiento de grandes porciones de población de zonas rurales a estas nuevas zonas urbanas provoca un aumento de la población en las ciudades y esto tiene efectos negativos en la calidad de vida de los habitantes urbanos, nuevos o no, sensibles a la calidad del aire (Powe \& Willis, 2004) y al calor intenso (Sánchez González, 2015).

En este contexto, los parques urbanos desempeñan un papel importante en la mejora de las condiciones de vida de los habitantes (Wiesztort, 2015) a través de sus servicios ecosistémicos (mejora de 
la calidad del aire, disminución de la isla de calor, oferta de zonas de sombra, paisaje verde y estética) (Wiesztort, 2018).

Numerosos estudios y encuestas de poblaciones urbanas han demostrado que los habitantes urbanos tienen una gran necesidad de naturaleza. Consideran que esto es algo que falta cada vez más en las ciudades y se quejan de la fuerte artificialización de estas (revestimientos de hormigón, piedra y ausencia de vegetación).

Las ciudades atraen a las personas que desean mejorar sus condiciones de vida y los antiguos habitantes de las zonas rurales sienten una nostalgia por el campo y los espacios naturales, y desean vivir esa experiencia para intentar escapar momentáneamente del ambiente asfixiante de las grandes ciudades. Desde hace algunas décadas, los planificadores han tendido a cubrir con cemento o construir sobre todos los espacios (carreteras asfaltadas, plazas artificiales y grandes aparcamientos). La densidad urbana sigue aumentando y muchos espacios abiertos están siendo construidos progresivamente.

Como consecuencia de lo anterior, la ciudad se percibe como un espacio frío y la ausencia general de naturaleza en la ciudad crea una carencia sentida por la población (Coupleux et al., 2008), que expresa un malestar.

\subsection{Un malestar en la ciudad}

La mitad de la población mundial vive en ciudades, así que el ser humano se convirtió en un homo urbanus, para tomar la expresión de Jeremy Rifkin (2000). Viviendo fuera de los espacios naturales, este homo urbanus aspira a recuperar ciertos elementos naturales. Entonces, busca "disponer de las amenidades de la ciudad, satisfaciendo al mismo tiempo su deseo de naturaleza y de tranquilidad" (Bourdeau-Lepage et al., 2012). Este homo urbanus se convierte en un homo qualitus al menos en las sociedades occidentales, es decir, un hombre que no solo busca su bienestar material e inmaterial, pero hace de la satisfacción de su deseo de naturaleza y de la preservación de su medio ambiente un elemento de su bienestar (Bourdeau-Lepage $\&$ Vidal, 2013). Por ejemplo, las tres cuartas partes de los franceses van a un parque más o menos a diario (Union Nationale des Entrepreneurs du Paysage [Unep] \& Ipsos, 2008). Para los franceses, el jardín privado o público sería uno de los primeros elementos que les permitirían mejorar su nivel de bienestar en la ciudad. Ahora, en la mayoría de las grandes ciudades del mundo los jardines privados representan la parte más importante de espacios de naturaleza urbana. Espacios que son accesibles únicamente por sus propietarios. Así, existe una desigualdad de acceso a la naturaleza urbana.

Se observa que el bienestar de la población urbana pasa por su proximidad a la naturaleza. La encuesta de Unep e Ipsos de 2013 revela también que la naturaleza para el 55,7\% de los encuestados, que viven o no en apartamentos, corresponde a una necesidad vital (al igual que la alimentación), en particular en forma de espacios verdes. La encuesta muestra a través de la pregunta “¿para ustedes, el contacto diario con la vegetación es muy importante, importante, poco importante o no importante?" que la gran mayoría de los europeos opta por las dos primeras respuestas.

¡La presencia de la naturaleza es esencial, vital! Es beneficiosa para la salud de manera directa e indirecta por su simple vista, actúa sobre la moral (estrés, violencia), por su contacto (pasear por un 
bosque, un parque, apreciar el aire puro, la vegetación, cultivar...).

Varios trabajos de investigadores extranjeros ponen de manifiesto este hecho. Por ejemplo: Raanaas et al. (2011) demuestran que la presencia de plantas dentro de una estructura de convalecencia influye positivamente en el estado de salud de los pacientes. Qing Li, en 2010, expone el hecho de que un paseo por el corazón de un espacio boscoso tiene notables beneficios inmunitarios. Da como ejemplo el aumento de las células asesinas naturales frente a nuestro nivel de inmunidad, es decir, cómo el sistema inmunológico se activa, permitiendo luchar contra ciertas enfermedades. Maas et al., en 2009, también muestran los efectos de la presencia de espacios verdes cerca del domicilio sobre la salud.

La naturaleza también tiene beneficios indirectos. El investigador Roger Ulrich, en un artículo publicado en 1984 en la revista Science, abre el camino a muchas otras investigaciones. Muestra una recuperación más rápida después de una intervención quirúrgica para pacientes que tienen, desde su habitación, una vista sobre árboles. Bunn-Jin Park et al. (2009a) exponen el hecho de que el lugar donde practicamos actividad física tiene efectos diferenciados sobre las personas. Así, un paseo en el bosque tendría efectos más positivos que en zonas urbanas.

De estos múltiples estudios se puede deducir que la ausencia de naturaleza puede tener efectos negativos en la salud mental y física, así como en el bienestar general de las personas. La obra de Boutefeu (2002) revela que cuando los urbanos salen del centro de la ciudad para vivir en el periurbano buscan una mejor calidad de vida, una casa con jardín individual, protegida de los ruidos exteriores y de las molestias urbanas, pero también el mantenimiento de un vínculo con la naturaleza. Cuando los habitantes de las zonas urbanas no tienen la posibilidad de vivir en las zonas periurbanas o rurales, se mueven allí para satisfacer sus necesidades (los fines de semana). Los espacios naturales en las zonas periurbanas son muy importantes.

\section{La zona periurbana, un espacio clave en los proyectos de renaturación de los territorios \\ urbanos}

Las ciudades son demasiado densas para reinsertar suficientes espacios que respondan a la demanda social y, a su vez, existen múltiples retos que estas plantean cuando se habla de los servicios ecosistémicos de la naturaleza. Las primeras políticas proponían utilizar los periféricos como soporte de proyectos medioambientales.

\subsection{Los cinturones verdes}

La necesidad de que las comunidades limiten la expansión urbana es cada vez más urgente. Los numerosos problemas que este fenómeno causa (alargamiento de las distancias recorridas, pérdida de tierras agrícolas, destrucción de los medios naturales y de la biodiversidad, entre otros) impulsan a la reflexión y a la acción. Es necesario limitar las posibilidades de urbanización en el espacio, limitar el perímetro de urbanización y, eventualmente, definir zonas prioritarias de ordenación.

Al principio de los años 1950, una solución aparece: el cinturón verde. Su objetivo era rodear la ciudad para contener su expansión y al mismo tiempo 
ofrecer espacios recreativos a los urbanos. Es un conjunto de espacios naturales protegidos conectados por corredores de vocación ecológica o recreativa en la zona periurbana que a veces penetran al interior de la metrópoli. La finalidad es ofrecer una mejor calidad de vida urbana y periurbana a los habitantes.

El concepto, creado para controlar la expansión de algunas ciudades, ha surgido en el Reino Unido (en Londres), donde se habla del Green Belt y, a su vez, en Canadá (en Ottawa, Toronto): el cinturón verde. En Francia, numerosas metrópolis han establecido también cinturones verdes: Rennes, Strasbourg, París y Lille.

El Green Belt de Londres fue la primera aplicación concreta (introducida entre los años 1930 y 1950). Este concepto tan evocador se repitió posteriormente con frecuencia, de manera más o menos rigurosa y eficaz. La idea básica es sencilla: crear una zona de contención compuesta por tierras agrícolas o medios naturales. Los objetivos asociados son múltiples:

- Preservar el patrimonio rural y el acceso a la naturaleza para los habitantes de las ciudades (en particular con fines recreativos).

- Beneficiarse de los efectos favorables de la proximidad de espacios verdes (calidad del aire, regulación climática, estética, etc.).

- Limitar la dispersión urbana y favorecer una utilización óptima del territorio.

- Garantizar la capacidad de producción de alimentos cerca de la zona urbana.

- Conservar la biodiversidad.
Sobre el papel, la implantación de un cinturón verde es muy atractiva, pero en la realidad los pocos experimentos de cinturones verdes son raros y sus éxitos son muy discutidos. Las razones: 1) la implantación de un cinturón verde debe ir acompañada de reglamentación en urbanismo y 2) se requiere un excelente control de las tierras designadas o el establecimiento de zonas no edificables definitivas.

Si bien los cinturones verdes ofrecen nuevos espacios de naturaleza que pueden frecuentar los habitantes de la ciudad, se ha comprobado que el establecimiento de una zona de amortiguación no urbanizable puede frenar la expansión urbana, pero solo temporalmente. Se habla de leapfrogging ${ }^{2}$. Sin otras medidas que orienten la forma en que se desarrolla la ciudad dentro del cinturón y la forma en que se desarrollan los municipios del exterior, el esfuerzo es inútil (reglamentación urbana estricta). Este problema se puso de manifiesto en el caso del cinturón verde de Ottawa, creado en 1950. El cinturón de vegetación, pensado en gran parte en expropiaciones y adquisiciones de tierras por la Comisión de la Capital Nacional, consta de más de 20.000 hectáreas de tierras naturales y agrícolas. Pero Ottawa ha conocido un crecimiento (demográfico y de su superficie) muy superior a lo previsto y cuenta actualmente con alrededor de 895.000 habitantes en una superficie de 279.600 hectáreas (Figura 1).

El cinturón de vegetación no permitió limitar la expansión urbana como se deseaba (expansión urbana más allá y en el cinturón verde). Sin embargo, la existencia de esta banda natural constituye un activo para la imagen de la capital cana-

2 El leapfrogging es un fenómeno de cruce. La ciudad se extiende más allá del cinturón verde. 


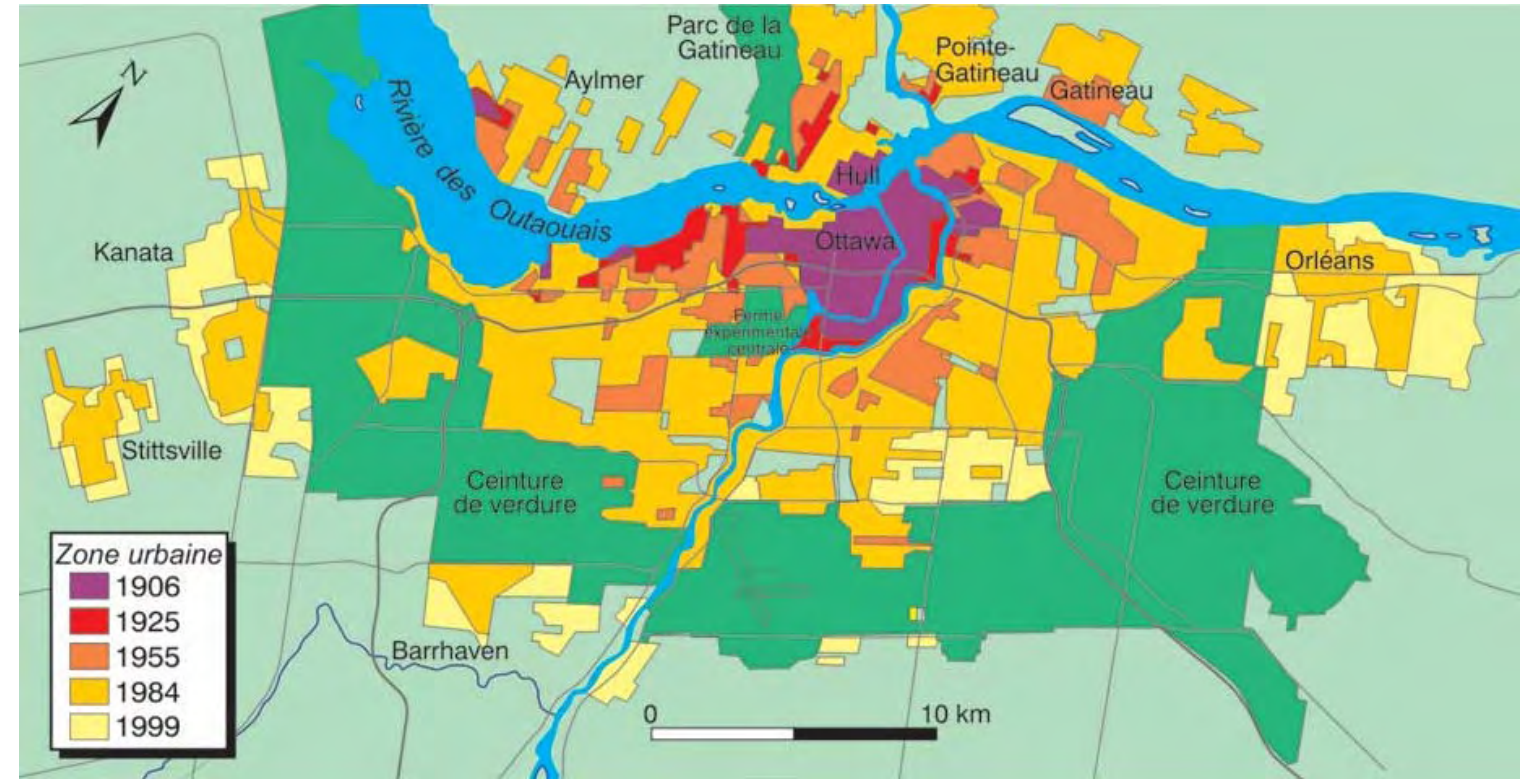

Figura 1. Cinturón verde en Ottawa

Fuente: Canadian Geoscience Education Network (2019).

diense y ofrece espacios agrícolas y naturales al corazón de la ciudad.

En otras partes del mundo, en particular en Londres o incluso en Toronto, las opiniones sobre los cinturones verdes son dispares. Entonces, ¿cómo se pueden satisfacer las necesidades de naturaleza de los ciudadanos?

\subsection{Parques lineales periféricos}

Hoy, los parques lineales están pensados tanto para responder a los retos ambientales como a los sociales. Ofrecen espacios de recreo (paseos, salidas naturalistas, senderismo, bicicleta, deportes acuáticos, juegos para niños, etc.) y son muy frecuentados por los ciudadanos (Silva Correia et al., 2010). Así, contribuyen al bienestar de las poblaciones que los visitan (Park et al., 2009a; Li, 2010).
Estos parques lineales, infraestructuras verdes o trama verde y azul, según cada país (Firehock, 2010; Pellegrino \& Moura, 2017), se crean sobre lineales vacíos a través de las ciudades densas. Por ejemplo: una antigua vía férrea, riberas de ríos o bajadas de carretera (Wiesztort, 2015).

Originalmente, estos parques lineales debían responder a objetivos ecológicos. Se pretende crear corredores biológicos que faciliten la migración de las especies (Benedict \& McMahon, 2006). Estos corredores podrán apoyarse en espacios naturales situados en la zona periurbana, es decir, alrededor de la ciudad densa, como el cinturón verde de Ottawa (Figura 1). El esquema se presenta en la Figura 2. 


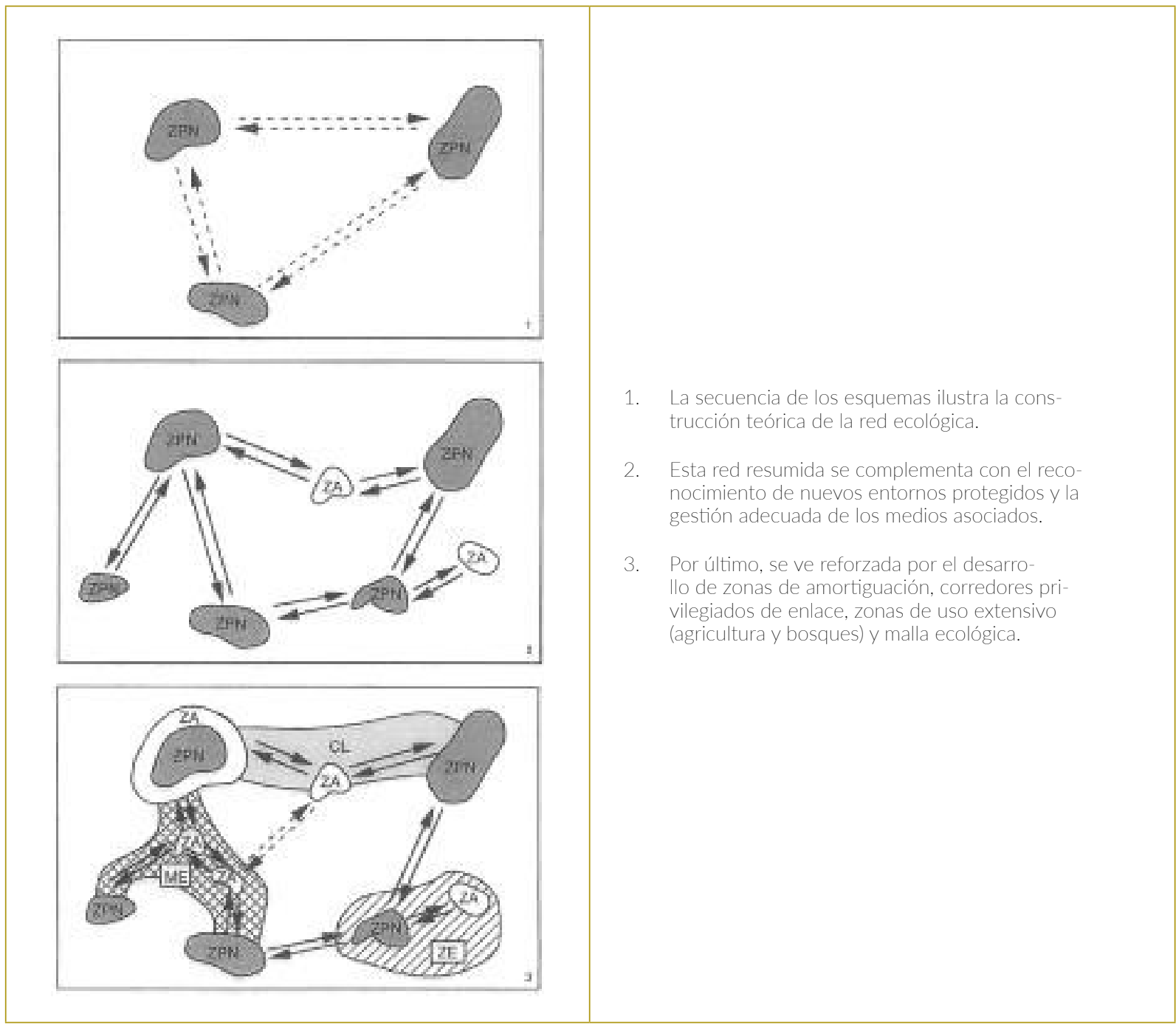

Figura 2. La red ecológica y la zonificación del espacio: esta red ecológica coherente y funcional responde a los objetivos de mantener la biodiversidad y la conservación de la naturaleza en torno a una organización espacial apropiada

Leyenda: ZPN: zonas de protección de la naturaleza; ZA: zonas asociadas; CL: corredor de enlace; ZE: zonas de uso extensivo; ME: malla ecológica que refuerza la red.

Fuente: elaboración propia con base en Melin (1997).

El reto social también es importante (Malard, 2002). Hay que responder a la demanda social de espacios de naturaleza practicables. Los habitantes quieren poder practicar actividades de ocio (juegos para niños, paseos, actividades deportivas, asistir a eventos culturales, comer, beber, picnic, etc.). La linealidad es una solución, ya que la presión inmobiliaria es demasiado fuerte y solo están disponibles unas pocas líneas que han perdido sus vocaciones primarias. Además, esta linealidad 
permite una mayor accesibilidad. El parque lineal puede atravesar varios barrios y convertirse así en un parque de proximidad para todos estos habitantes. Por proximidad se entiende un máximo de 200 metros de radiación alrededor del domicilio. En este radio, estamos en un espacio de proximidad al que puede tener acceso una persona mayor a pie todos los días (García-Ballesteros \& Jiménez, 2016; Sánchez González, 2015).

A través dos ejemplos reales, vamos a observar y estudiar la concepción de dos parques lineales: uno en Francia, en Lille, y un otro en Brasil, en Río de Janeiro. En Francia, los proyectos son recientes y responden a numerosos textos y leyes a nivel internacional y nacional. El interés por la naturaleza en las ciudades ha adquirido particular importancia en Francia con la política de la Agenda 21 y de la Cumbre de Río en 1992 y, posteriormente, de las Grenelles de 1'Environnement en 2009 y 2010. En Francia, se hablará de trama verde y azul y van a pensar estos parques lineales en las riberas de los ríos.

Después de haber ignorado durante mucho tiempo sus ríos, transformándolos en vías de circulación, aparcamientos o almacenes, la mayoría de las grandes ciudades francesas e incluso europeas crean grandes operaciones de reconversión. Hoy se habla de "reconquista" (Ghorra-Gobin, 2015; Pénet, 2004) o de "reconciliación" (Bonin, 2007). Con el tiempo, los residentes urbanos habían perdido el contacto directo con el río (Landrin, 2012). La visión del río ha ido cambiando gradualmente: ya no está dominada únicamente por el temor a las inundaciones en las grandes ciudades (Wiesztort, 2015). El proyecto de trama verde y azul (TVB) de la metrópoli de Lille es un buen ejemplo que vamos a presentar.
En Brasil, los años noventa son importantes en el proceso de integración de las políticas ambientales urbanas. En 1992, la Declaración de Río de las Naciones Unidas ofreció importantes orientaciones para una política ambiental. Se han creado instrumentos normativos como la Ley de la Naturaleza en 1998, el establecimiento del Sistema Nacional de Unidades de Conservación (SNUC) en 2000 y la Agenda 21 de 2002 para promover el desarrollo sostenible.

Lo anterior ofrece un marco favorable para el desarrollo de parques lineales como instrumento útil para la planificación urbana. Sin embargo, el proceso de implantación de los parques lineales en Brasil sigue siendo reciente debido a las numerosas dificultades (retos económicos, sociales, medioambientales, falta de espacio disponible, presión inmobiliaria, etc.) con que se ha tropezado para pasar del proyecto a la realización. Un proyecto debe adaptarse a su territorio y tomar en cuenta cada de sus especificidades.

Además, dichos parques existen en los planes de urbanismo y en las leyes municipales de parcelación del suelo urbano, pero muchos no se crean o se crean parcialmente ya que, en general, los espacios públicos y las áreas verdes no son una prioridad en la distribución presupuestaria de los municipios. En la mayoría de los casos, se consideran espacios restringidos, como franjas de servidumbre a lo largo de las líneas de transmisión de energía o del ferrocarril. El Parque Madureira, ubicado en las afueras de Río de Janeiro, es un ejemplo de las funciones asignadas a este tipo de parques y también revela los conflictos existentes en el proceso de implantación y gestión. 


\section{Estudio de caso en Brasil y}

\section{Francia}

\subsection{La zona periurbana de Lille responde al bienestar de los \\ metropolitanos a través de su oferta} de parques lineales

Lille es una metrópoli ubicada en la región de Hauts de France. Se trata de una región que se ha dedicado rápidamente al desarrollo sostenible $\mathrm{y}$, en particular, a las políticas de reinserción de naturaleza en el territorio. Han sido precursores de muchas y diversas políticas de desarrollo sostenible en Francia y también un modelo para las otras regiones.

A partir de 1992, se han concebido coladas verdes para recorrer el territorio regional. Una colada verde es un espacio verde lineal acondicionado que atraviesa la ciudad o un barrio y está protegido en el marco de un plan de urbanización. Puede tener una vocación de corredor ecológico o inscribirse en una red de desplazamientos suaves ( $\sin$ motor). Estos proyectos fueron motivados por la constatación de que la importante fragmentación del territorio provocaba el fraccionamiento y la fragilización de las poblaciones vegetales y animales, incluidas las especies ordinarias. Esta fragmentación se debe también a los eventos históricos que han destruido los espacios naturales (las guerras y la intensa actividad industrial hullera, entre otros).

Los espacios rurales de proximidad, los cursos de agua, los ríos y las zonas urbanas deben volver a ser espacios de vida para la naturaleza. A escala de las ciudades especialmente densas como la metrópoli de Lille, es necesario administrar de forma sostenible el patrimonio natural existente o rehabilitarlo si este se reduce o no está presente. A esto se añade también la función social. Gracias a estos espacios lineales, la trama verde y azul se ha convertido en el soporte de senderos en las riberas de los ríos y los antiguos cavaliers (vías férreas del período industrial hullero que permiten transportar materiales y carbón). Al final de los años sesenta, a escala de la metrópoli de Lille, el Estado lanzó el proyecto de un gran equipamiento de ocio para contribuir a la rehabilitación del paisaje entre la metrópoli de Lille y la aglomeración de Lens. Este proyecto de mil hectáreas también tenía por objeto proteger los campos que captaban un tercio del recurso hídrico de la metrópoli. Los vecinos, en particular los agricultores, rechazaron el proyecto, que era demasiado ambicioso y requería demasiado terreno, así que la iniciativa fue abandonada y aparecería de nuevo 30 años más tarde, en un nuevo contexto. En efecto, el desarrollo de la metrópoli de Lille ha puesto de manifiesto un déficit de espacios recreativos y la agricultura se mantiene difícilmente en zonas periféricas en las que se entremezclan vida urbana y vida rural. Las mentalidades han cambiado, las preocupaciones medioambientales y el entorno de vida son cada vez más importantes y el recurso hídrico sigue siendo frágil. En la década de los años noventa, la Comunidad Urbana de la Metrópoli de Lille y las ciudades de Houplin-Ancoisne, Santes, Wavrin, Don, Haubourdin y Seclin piensan de nuevo el proyecto con tres arquitectos paisajísticos: Jacques Simon (Gran Premio del Paisaje 1990), Jean-Noël Capart e Yves Hubert, de Joining Nature and Cities (JNC) International (grandes ganadores del concurso internacional). Así, en la zona periurbana próxima se pensará el Parque lineal Deûle (Figura 3). 


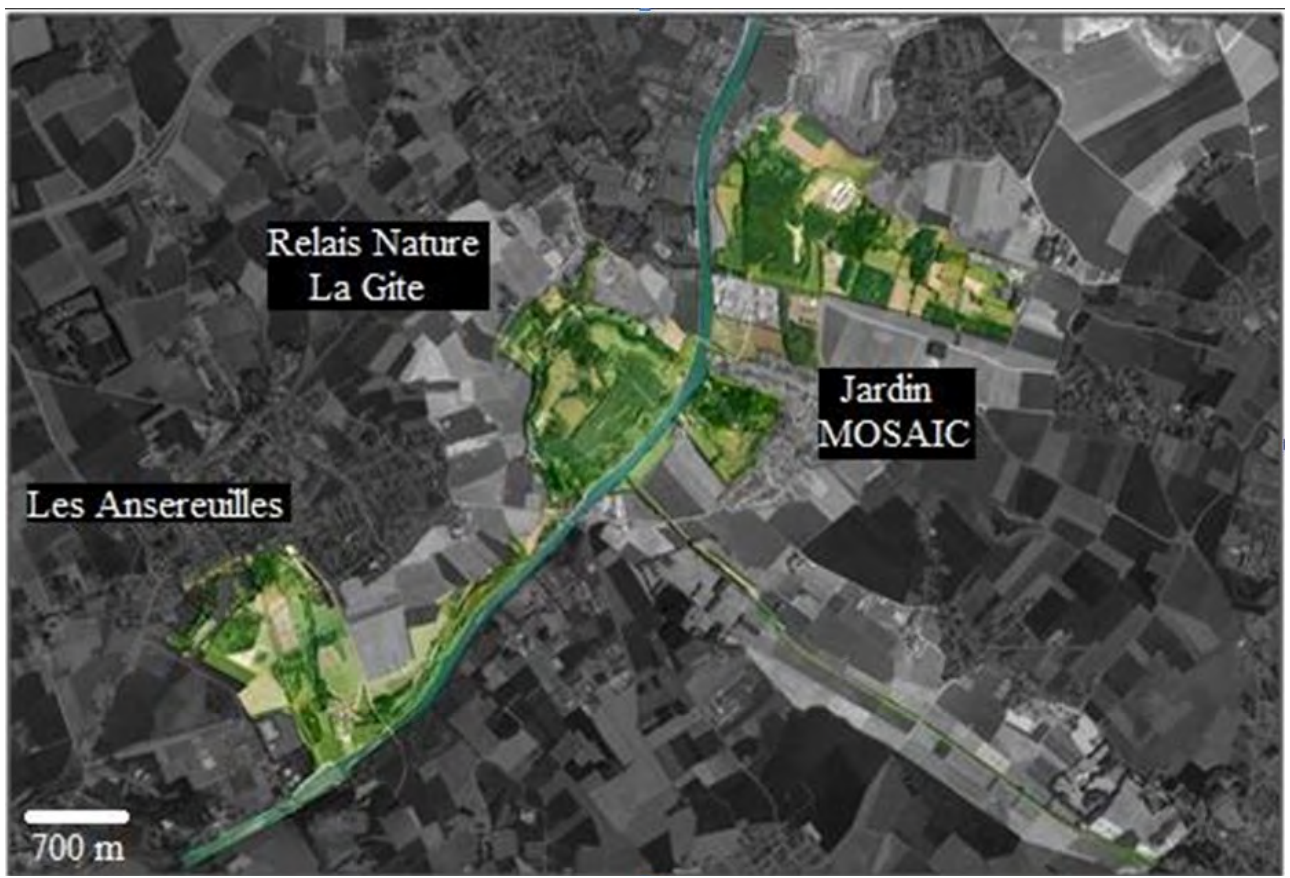

Figura 3. Vista aérea del trazado territorial de los jardines del Parque Deûle

Fuente: elaboración propia (2018).

El Parque Deûle es original porque tiene forma de rosario y se compone de varias entidades que bordean el río Deûle. Se extiende sobre seis municipios. Para los diseñadores, había que interesarse por todos los espacios de la urbanización tradicional. En febrero de 1999, estas 277 hectáreas fueron declaradas de utilidad pública y se iniciaron les primeras obras. El Parque Deûle es un parque en red (Figura 4) de unos treinta kilómetros que conecta espacios periurbanos, postindustriales y agrícolas a lo largo del antiguo canal industrial de Deûle, antaño uno de los más contaminados de Francia. Hoy, el parque se extiende sobre 300 hectáreas. Los retos del proyecto son importantes: 1) la protección de los recursos hídricos, 2) la protección y estructuración de los ecosistemas ricos (praderas húmedas, zonas pantanosas) y 3) la reconquista de la imagen y de la calidad paisajística.
Esta zona periurbana se había transformado en una ciudad dormitorio. Así, la idea era que el parque se convirtiera en un vínculo entre los territorios habitados y evitara la conurbación Lille-Lens. Una conurbación es un conjunto urbano constituido por varios núcleos urbanos (Lille y Lens en ese caso) cuyos suburbios acaban por unirse. Además, se han realizado obras considerables para limpiar numerosos espacios industriales (vertederos y zonas baldías de las vías navegables francesas) para transformar el suelo, declinar los medios húmedos y plantar miles de árboles. El sitio de la Gîte se ha convertido en un parque de interpretación de la fauna y la flora (Figura 4). El Parque de Santes revela un mosaico de paisajes: un valle semiforestado, medio prado, cuencas de agua y humedales (Figura 4). Por último, los espacios de ocio están estrechamente entrelazados en los territorios agrícolas gracias a los senderos. 

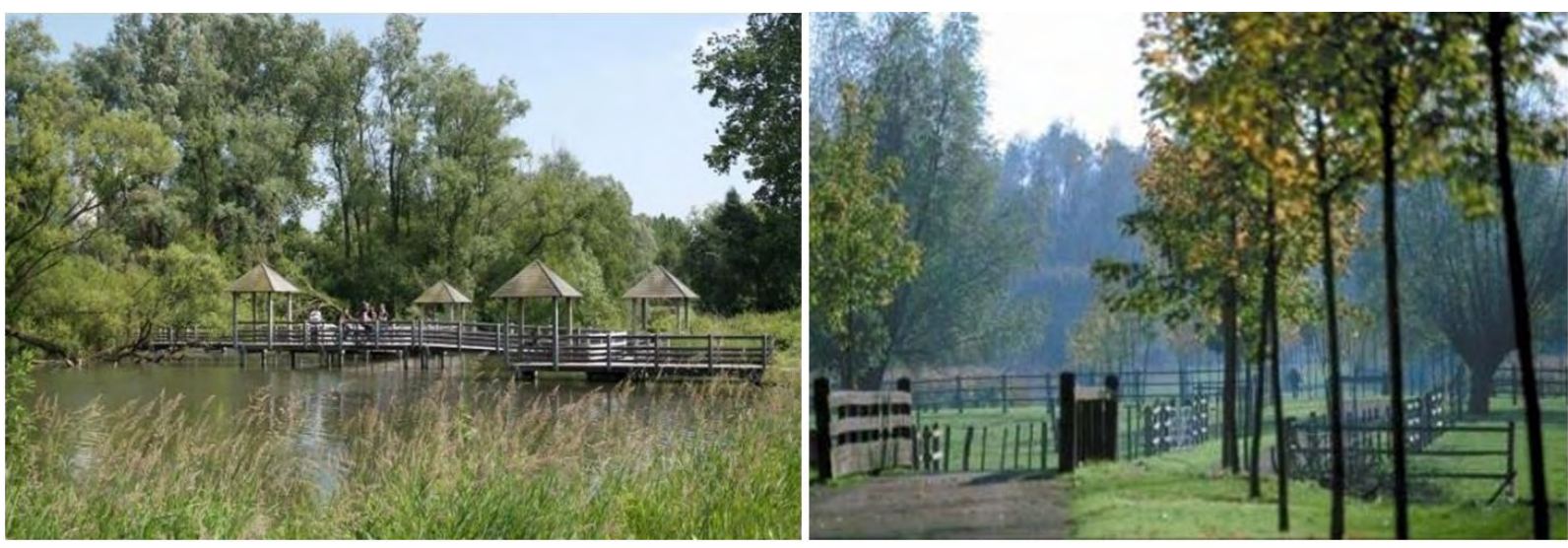

Figura 4. Parque La Gîte

Fuente: fotografías tomadas por la autora (2015).

El parque está concebido como un espacio de encuentro y de cultura, donde además se permite el desarrollo de actividades recreativas y existen numerosas animaciones y manifestaciones. Por ejemplo, en el Parque Deûlese se encuentra el Jardín Mosaic o Jardín de las Culturas (Figura 5), que representa la diversidad cultural de la población metropolitana de Lille. Los paisajistas trabajaron con las comunidades que componen la aglomeración y con los artistas en torno a la pregunta: “¿La gente tiene sueños de jardines diferentes según su cultura?”. En 33 hectáreas, se han diseñado diez jardines contemporáneos (que representan simbólicamente diez jardines de sueños de individuos de diversas culturas: mediterránea, inglesa, asiática, etc.), un estanque y un bosque. Todos los domingos por la tarde, el Parque Mosaic ofrece espectáculos y animaciones de todo tipo: música, teatro, talleres, visitas guiadas.

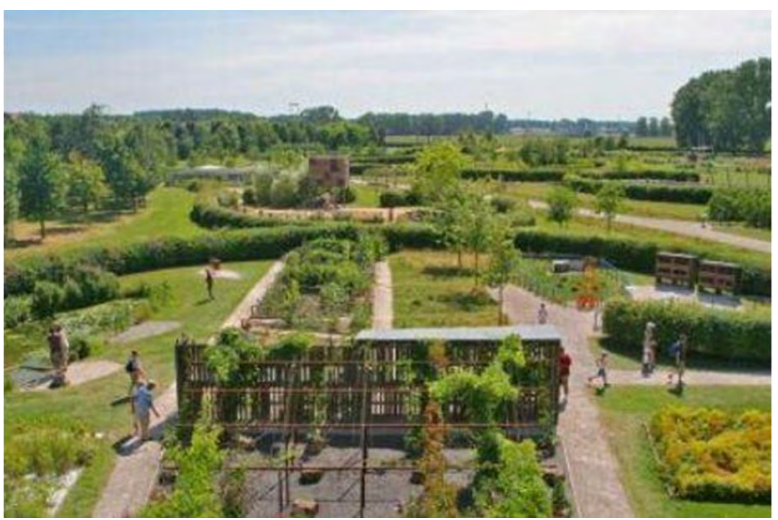

Figura 5. Parque Mosaic

Fuente: fotografías tomadas por la autora (2018).

En 2006, el Parque Deûle recibió el Premio Nacional del Paisaje y en 2009 el Premio del Paisaje del Consejo de Europa. También se ha convertido en un referente como parque periurbano de una gran metrópoli. Hoy, el parque forma parte de un proyecto más amplio denominado Objetivo Metropolitano Verde, que tiene por objeto realizar una corona verde transfronteriza que debería desplegarse dentro de diez años en unas 10.000 hectáreas. 


\subsection{El Parque Madureira y las} reservas naturales de Río de Janeiro aportan naturaleza a los urbanos

En Brasil se habla de la infraestructura verde (IV) que, al igual que la trama verde y azul en Francia, debe servir para el abastecimiento de los servicios ecosistémicos y ofrecer más de una función en un mismo espacio (Pellegrino \& Moura, 2017). Participa en la mejora de la calidad del aire y de los suelos, del microclima, trata y protege los recursos hídricos y ofrece hábitats para la fauna, así como espacios de ocio y socialización.

Los parques lineales en las ciudades brasileñas tienen tres retos principales: la protección de los cursos de agua, el diseño de nuevos corredores ecológicos y la creación de espacios de ocio para los urbanos.

Según Mayorga Mora (2013), el parque lineal es una solución para los problemas de drenaje en la ciudad, al tiempo que promueve la protección de estos cursos de agua. También es importante el compromiso de establecer corredores ecológicos. Bonzi (2015) explica que deben permitir la integración paisajística, conectar físicamente los parches de vegetación y garantizar el flujo de gente entre poblaciones aisladas.

Aunque la ciudad de Río de Janeiro es conocida por su paisaje y su relación con la naturaleza, su distribución de zonas verdes y espacios públicos es desigual. En las zonas periurbanas, los espacios libres son pocos y la densificación de la ciudad se "come" las áreas de preservación medioambiental.

El Parque Madureira, al norte de Río de Janeiro, fue creado para paliar esta situación. Según Soares (2015), el terreno elegido es el resultado de la compactación de líneas de transmisión de energía de la empresa Light. Estas líneas ocupan actualmente una franja de cincuenta metros en el corazón de grandes ejes de comunicación (Figura 6). El barrio de Madureira, donde se encuentra el parque, es considerado uno de los mayores centros de comercio del suburbio de Río de Janeiro (Soares, 2015). Su ubicación central, entre dos estaciones ferroviarias (Rocha Miranda y Honório Gurgel), es atractiva. Hay numerosos comercios y actividades culturales, como las escuelas de samba Portela e Imperio Serrano. Es también una zona residencial con una población familiar que desea espacios de naturaleza que puedan usar. Según el Plan Director decenal, hay una carencia de zonas verdes (menos de $1 \mathrm{~m}^{2}$ por habitante), espacios culturales, deportivos, de ocio y de contemplación. En efecto, en esta zona geográfica los espacios de naturaleza transitables son insuficientes y solo unos pocos árboles de pequeño y mediano tamaño ofrecen vegetación al barrio.

En cuanto a la implantación e integración del proyecto, no se tuvo en cuenta lo existente, como las actividades económicas (comercios locales), culturales y sociales del barrio. Esto es un problema cuando sabemos que los barrios próximos al parque tienen una alta tasa de violencia y delincuencia que ha aumentado desde la creación del parque. Con el Parque Madureira, la ciudad tenía la ambición de ofrecer diversos espacios para el ocio y así responder a las expectativas de los habitantes. En Brasil, los discursos en favor de la promoción de la sostenibilidad en las zonas urbanas a menudo se reducen a la idea de la calidad del espacio público con una orientación principal (el deporte y el ocio) y se utilizan como instrumentos para hacer frente a los problemas de violencia y promover la educación. 


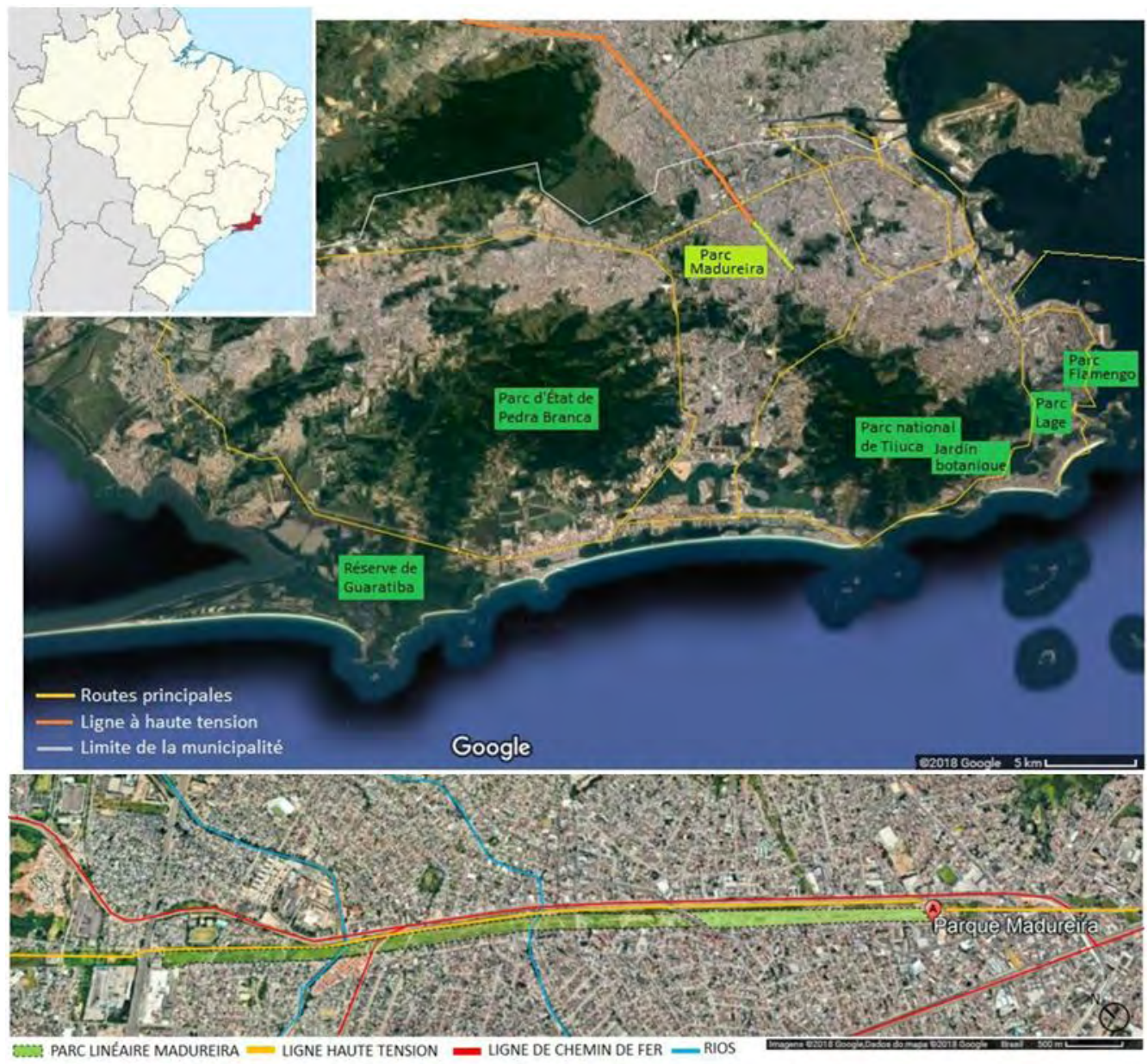

Figura 6. Mapas de ubicación del Parque Madureira en Río de Janeiro

Fuente: Amorim y Wiesztort (2018).

Sin embargo, en el proceso de implantación del Parque Madureira no ha habido consideración para los residentes del sector elegido. En Brasil, cuando los parques lineales se instalan en zonas de gran densidad residencial como el barrio de Madureira, el proceso de expropiación es común (633 habitantes fueron expropiados y 264 realojados gracias al programa brasileño Mi casa Mi vida, pero en localidades alejadas del parque [Silva, 2013]). Para que un proyecto tenga éxito, debe ser aceptado por la población y, para que sea así, es preferible que se les tenga en cuenta.
Hoy, el Parque Madureira es el tercer parque más grande de la ciudad, después del Aterro do Flamengo y el de la Quinta da Boa Vista. Fue construido en varias etapas. En 2010 se realizaron las primeras obras en el barrio de Madureira para diseñar una infraestructura verde lineal de $109.000 \mathrm{~m}^{2}$ (Figura 6). La segunda fase corresponde a la ampliación del parque existente y se llevó a cabo entre 2014 y 2016 con un objetivo de 255.000 $\mathrm{m}^{2}$ a través de seis barrios (Figura 6).

En 2016, el corazón del Parque Madureira se pensó como villa olímpica. A diferencia de otras estructu- 
ras construidas para los Juegos Olímpicos, el Parque Madureira ha evolucionado y no ha perdido su popularidad, porque es cada vez más frecuentado. Actualmente, el parque está compuesto por instalaciones deportivas (carriles bici, juegos, equipa- mientos deportivos y un skatepark), de ocio y cultura (parques infantiles, playa artificial, cascadas de agua, lugar para la práctica de la samba, etc.). El parque se divide en cuatro sectores (Figura 7).

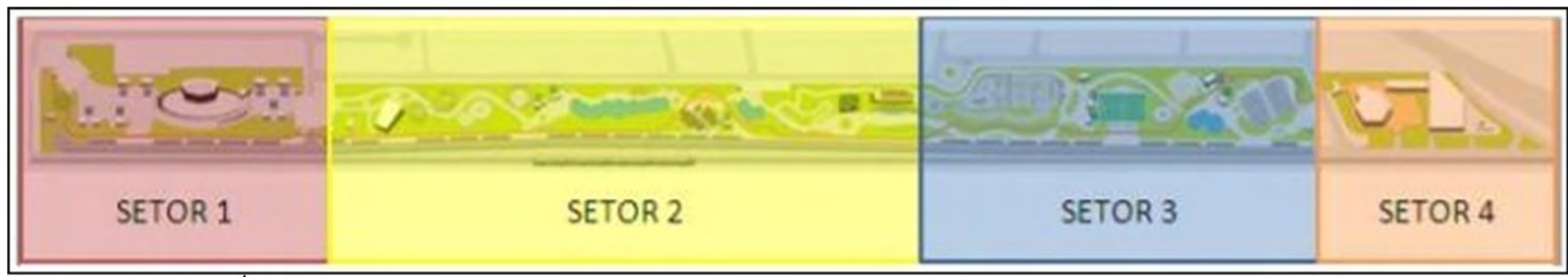

Figura 7. Los cuatro sectores del Parque Madureira

Fuente: Chagas Bonelli y Romanel (2012).

- Sector 1: cerca de la zona comercial, este sector llamado Praçado Samba está destinado a eventos musicales y culturales. Hay muchos quioscos para alimentarse.

- Sector 2: el parque contemplativo. Es un espacio de relajación y contemplación. Allí se encuentran kioscos de información y también bicicletas para pasear por el parque. Hay un jardín de los sentidos que permite acercar la vegetación a través de los cuatro sentidos. Existe también un jardín botánico que permite identificar las especies y sus características. En este sector se concede un lugar especial a las personas mayores. Se han acentuado la accesibilidad y el confort.

- Sector 3: se dedica a las actividades deportivas. Está el segundo mayor parque de skatepark del país, campos de múltiples deportes, fútbol, voleibol de playa, etc. También hay un lago de $387 \mathrm{~m}^{2}$.
- Sector 4: la Carioca Arena es un lugar de conciertos y teatro con capacidad para unas 330 personas dentro y más de 1.500 personas en zona exterior.

Este parque es un éxito social a escala de la metrópoli. La presencia de las dos estaciones ferroviarias y de las paradas de autobuses permite una gran accesibilidad y el parque responde a la demanda de los urbanos por espacios de ocio, pero no a escala de los barrios donde está implantado. Es cierto que los habitantes lo frecuentan y lo aprecian, pero recordemos que, para la implantación del proyecto, no se tuvo en cuenta lo existente. Esto es un problema cuando sabemos que tiene una alta tasa de violencia y delincuencia. Las expropiaciones en origen también son nefastas en el proceso de apropiación del parque. Así, parece que las diferentes partes del Parque Madureira responden a las necesidades de la población, pero las expropiaciones también han 
agravado situaciones como la exclusión social. La descripción de los pasos permite destacar la creación de espacios de ocio para los ciudadanos que carecen de naturaleza... pero la preocupación sigue siendo ¿cómo el parque protege los ríos? ¿Y cómo su diseño crea nuevos corredores ecológicos? Hoy en día, estos arreglos son inexistentes.

\section{Conclusión}

Inspirados ambos por la Declaración de Río de 1992, los Estados, motivados por los retos medioambientales, han establecido textos y leyes que tienen por objeto motivar la acción en su territorio. La reinserción de la naturaleza en la ciudad y en particular el proceso de implantación de parques lineales, tanto en Brasil como en Francia, es reciente. En Brasil, la mayoría de estos parques no son creados o solo son creados parcialmente. Los espacios públicos y las zonas verdes no aparecen como una prioridad en la distribución financiera de los municipios. Esto se puede generalizar a muchos países. En Francia, los lineales con vocación ecológica no son muchos. Muchos son parques ordinarios con una geometría particular (lineal). No encontramos aquí los retos específicos de la trama verde y azul o de la infraestructura verde, en particular, ecológica.

Los dos parques lineales presentados están ubicados en zonas periurbanas de dos grandes metrópolis. Ambos responden a una fuerte demanda de naturaleza en la ciudad por parte de los urbanos.
Tanto la metrópolis de Lille como la de Río de Janeiro son muy densas y sufren una fuerte presión inmobiliaria. Ambas metrópolis están cerca de espacios naturales como las reservas naturales de Río de Janeiro o los parques naturales regionales (PNR) de Lille, pero las reservas no son espacios que respondan completamente a la solicitud social. Los habitantes de las ciudades quieren espacios de naturaleza practicables, equipados para realizar actividades deportivas, usar juegos para niños, senderos para pasear, hacer picnic, etc.

Por el contrario, el Parque Deûle y el Parque Madureira responden perfectamente a esta solicitud, ya que ofrecen espacios verdes que pueden usar gracias a los espacios recreativos y sus equipamientos (mobiliario de confort, aseos, etc.), que permiten pasar un largo tiempo, medio día o un día, en familia o con amigos.

Sin embargo, hay que terminar con una crítica negativa: el Parque Madureira no responde en ningún caso a los retos ecológicos que plantea una infraestructura verde. Como hemos explicado, en Brasil los discursos a favor de la promoción de la sostenibilidad y del medio ambiente en las zonas urbanas a menudo se reducen a la idea de la calidad del espacio público, con su principal orientación hacia el deporte y el ocio. Por su parte, el Parque Deûle ofrece muchos espacios naturales (siega tardía, pradera húmeda, mar, etc.), pero menos equipos deportivos y permite a los ciudadanos reconectarse más con la naturaleza, como lo demuestran las fotografías de la Figura 5. 


\section{Referencias}

Amorim, N. \& Wiesztort, L. (2018). Les parcs linéaires comme infrastructures vertes ou trame verte et bleue répondant aux enjeux de la ville durable. Etude de cas et méthode comparative au Brésil et en France. Coloquio sobre cuestiones de ordenación y urbanismo: Naturaleza y ciudad: miradas cruzadas franco-lusófonas. APERAU \& programa franco-brasileño Attilio, Tours, 13-15 de junio.

Aragau, C. (2018). Le périurbain: un concept à l'épreuve des pratiques. Géoconfluences, abril. http://geoconfluences.ens-lyon.fr/informations-scientifiques/dossiersregionaux/france-espaces-ruraux-periurbains/articles-scientifiques/periurbainconcept-pratiques.

Benedict, M. A. \& McMahon, E. T. (2006). Green Infrastructure - linking landscapes an communities. Island Press.

Bilal, U., Díez J., Alfayate, S., Gullón, P., Del Cura, I., Escobar, F., Sandín, M., ... Franco, M. (2016). Population cardiovascular health and urban environments: The Heart Healthy Hoods exploratory study in Madrid, Spain. BMC Medical Research Methodology, 16(1), 104.

Björk, J., Albin, M., Grahn, P., Jacobsson, H., Ardö, J., Wadbro, J.\& Ostergren, P. (2008). Recreational values of the natural environment in relation to neighbourhood satisfaction, physical activity, obesity and wellbeing. Journal of Epidemiology \& Community Health, 62, 2e. http://dx.doi.org/10.1136/jech.2007.062414.

Bonin, S. (2007). Fleuves en ville : enjeux écologiques et projets urbains. Strates, 13. http:// journals.openedition.org/strates/5963.

Bonzi, R. S. (2015). Caminar sobre el agua Negro: infraestructura verde en zonas densamente pobladas. [Disertación de maestría, PPGAU-FAUUSP].

Bourdeau-Lepage, L., Langlois, W.\& Sable, T. (2012). La nature en ville. Espaces verts et bien-être. [Enquête menée auprès de 150 personnes à Lyon au printemps]. Centre de recherche en géographie et aménagement, Lyon : université Jean Moulin Lyon-3.

Bourdeau-Lepage, L. \& Vidal, R. (2013). Nature urbaine en débat : à quelle demande sociale répond la nature en ville? Le Déméter 2013, dossier Nature et agriculture dans la ville. Les nouveaux désirs des citadins s'imposent (pp. 195-210).

Boutefeu, E. (23 avril, 2002). Ne plus tenir la nature hors la ville. Revue Tech-Cités, 28.

Canadian Geoscience Education Network (2019). L'utilisation des terres. Géopanorama d'Ottawa et de Gatineau. https://fr.cgenarchive.org/ottawa-gatineau-landuse.html.

Chagas Bonelli, M. \& Romanel, C. (2012). Sustentabilidade em obra publicas: o caso do parque Madureira. En Anais do III Seminário de Pós-Graduação em Engenharia Urbana. http://www.eventos.uem.br/index.php/simpgeu/simpgeu/paper/view/988/743.

Cluzet, A. (2002). Au bonheur des villes. Editions de l'Aube.

Coupleux, S., Duhamel, S., Barthon, C., Delonnoy, M., Renard, J-P. \& Wiesztort, L. (2008). 
Attentes des personnes âgées en matière delogement. l'IFRESI et le bailleur Pas-deCalais Habitat.

Firehock, K. (2010). A short history of the term green infrastructure and selected literature. http://www.gicinc.org/PDFs/Gl\%2OHistory.pdf.

François-Melin, É. (1997). La problématique du réseau écologique. Bases théoriques et perspectives d'une stratégie écologique d'occupation et de gestion de l'espace. Actes du colloque international "Le réseau écologique", Arquennes, 1995. Travaux de la Conservation de la Nature, $n^{\circ} 18$, pp. 39-56.

García-Ballesteros, A. \& Jiménez, B. C. (2016). Envejecimiento y urbanización: implicaciones de dos procesos coincidentes. Investigaciones Geográficas, 89, 58-73.

Ghorra-Gobin, C. (2015). La dimension culturelle des espaces publics métropolitains. Tous Urbains, 12(4),6-7.

Gómez Mendoza, J. (2003). Ciudades desnaturalizadas y deslocalizadas. El Ecologista, 38, 37-45. https://www.ecologistasenaccion.org/7532/naturaleza-y-ciudad/.

Grahn, P. \& Stigsdotter, U. K. (2010). The relation between perceived sensory dimensions of urban green space and stress restoration. Land-scape \& Urban Planning, 94, 264275.

Landrin, S. (7 juillet, 2012). Les berges ramènent la nature au cœur des villes. Le Monde, p. 6.

Li, Q. (2010). Effect of forest bathing trips on human immune function. Environmental Health and Preventive Medicine, 15(1), 9-17.

Maas, J., van Dillen, S., Verheij, R. \& Groenewegen, P. (2009). Social contacts as a possible mechanism behind the relation between green space and health. Health \& Place, 15(2), 586-595.

MacKerron, G. \& Mourato, S. (2013). Happiness is greater in natural environments. Global Environmental Change, 23(5),992-1000.

Malard, V. (2002). La prise en compte de la nature dans l'aménagement urbain Trame verte de Saint Pierre des Corps. [Mémoire de recherche de Magistère d’Aménagement, Université de Tours].

Mayorga Mora, N. (2013). Experiencias de parques lineales en Brasil: espacios multifuncionales con potencial para brindar alternativas a problemas de drenaje y agua surbanas. Banco Interamericano de Desarrollo, nota técnica IDBTN-518. https:// publications.iadb.org/es/experiencias-de-parques-lineales-en-brasil-espaciosmultifuncionales-con-potencial-para-brindar.

Melé, P. (2004). Introduction: Conflits, territoires et action publique. En P. Melé, C. Larrue $\&$ M. Rosemberg (coords.), Conflits et territoires (pp. 1-19). Presses universitaires.

Park, B. J., Tsunetsugu, Y., Kasetani, T., Kagawa, T., \& Miyazaki, Y. (2009a). The physiological effects of Shinrin-yoku (taking in the forest atmosphere or forest bathing): evidence from field experiments in 24 forests across Japan. Environmental Health and Preventive Medicine, 15(1), 18-26. 
Park, B. J., Tsunetsugu, Y., Kasetani, T., Morikawa, T., Kagawa, T. \& Miyazaki, Y. (2009b). Physiological effects of forest recreation in a young conifer forest in Honokage Town, Japan. Silva Fennica, 43(2), 291-301.

Pellegrino, P. R. M. \& Moura, N. B. (2017). Estratégias para uma infraestrutura verde. Manole.

Pénet, M. (2004). La chanson de la Seine. Sociétés \& Représentations, 17, 51-66.

Powe, N. A. \& Willis, K. G. (2004). Mortality and morbidity benefits of air pollution (SO2 and PM10) absorption attributable to woodland in Britain. Journal of Environmental Management, 70, 119-128.

Raanaas, R. K., Patil, G. G. \& Hartig, T. (2011). Health benefits of a view of nature through the window: a quasi-experimental study of patients in a residential rehabilitation center. Clinical Rehabilitation, 26, 21-32.

Rifkin, J. (2000). L'Âge de l'accès. La Découverte et Syros.

Ruiz, J., Parra, E. \& López-Carr, D. (2015). Una visión geográfica de los parques urbanos de la ciudad de Tunja, Boyacá, Colombia. Perspectiva Geográfica, 20(2), 245-268.

Sánchez González, D. (2015). Ambiente físico-social y envejecimiento de la población desde la gerontología ambiental y geografía. Implicaciones socioespaciales en América Latina. Revista de Geografía Norte Grande, 60, 97-114.

Silva Correia, B., Corrêa da Silva, M. \& Magnabosco, M. (2010). Naturaleza y ocupación del espacio urbano. Los parques de la ciudad. Bitácora Urbano Territorial, 16(1), 107 120.

Silva, L. C. (2013). Ônus da construção de um espaço público de lazer no subúrbio carioca: remoções e valorização imobiliária associadas ao Parque Madureira. Anais do XV Enanpur, 15(1). anpur.org.br/ojs/index.php/anaisenanpur/article/view/271/263.

Soares, D. M. T. (2015). Parque Madureira: requalificação da paisagem urbana. [Disertación de Maestría en Ingeniería Urbana, Escola Politécnica da Universidade Federal do Rio de Janeiro].

Ulrich, R. (1984). View through a window may influence recovery from surgery. Science, 224, 420-421.

Union Nationale des Entrepreneurs du Paysage (Unep) \& Ipsos (2008). Les Espaces verts de demain. Usages et attentes des Français. Dossier de presse, sondage pour le compte de l'Unep. Unep.

Union Nationale des Entrepreneurs du Paysage (Unep) \& Ipsos (2013). Jardins et espaces verts de demain, l'exception culturelle française? Résultats de l'enquête Unep-Ipsos 2013. https://www.gre-mag.fr/site/wp-content/uploads/2014/12/dp_unep_ipsos_2013. pdf.

White, M. P., Alcock, I., Wheeler, B. W., \& Depledge, M. H. (2013). Would you be happier living in a greener urban area? A fixed-effects analysis of panel data. Psychological Science, 24(6), 920-928.

Wiesztort, L.(2011). La réinsertion de la nature en ville et le développement durable. Etudes de cas dans l'ancien bassin minier du Nord-Pas-de-Calais. [Thèse de doctorat, Université d'Artois]. 
Wiesztort, L. (2015). Les berges des fleuves ou cours d'eau traversant les métropoles comme moyen de réinsérer de la "nature" en ville ? Expériences de plusieurs métropoles françaises. Cuadernos Geográficos, 54(2), 124-150.

Wiesztort, L. (2018). Pratiques des personnes âgées dans les parcs de proximité. Etude de cas dans l'agglomération de Monterrey (Mexique). Les cahiers QuaMoTer, Ville inclusive - Ville genrée, pratiques des espaces publics, numéro de 2018. https:// quamoter.hypotheses.org/372. 